\title{
Ouverture de la Journée
}

\section{Philippe Caron}

\section{OpenEdition}

\section{Journals}

Édition électronique

URL : https://journals.openedition.org/rhcf/552

DOI : 10.4000/rhcf.552

Éditeur

Rails \& histoire

Édition imprimée

Date de publication : 1 novembre 2005

Pagination : 5-9

ISSN : 0996-9403

Référence électronique

Philippe Caron, «Ouverture de la Journée », Revue d'histoire des chemins de fer [En ligne], 32-33 | 2005, mis en ligne le 28 mars 2011, consulté le 22 avril 2022. URL : http://journals.openedition.org/rhcf/552 ; DOI : https://doi.org/10.4000/rhcf.552 


\title{
Philippe CARON
}

\section{Ouverture de la Journée}

\author{
Monsieur le Président, \\ Mesdames et Messieurs,
}

Vous me permettrez d'excuser Monsieur le Ministre de l'Écologie et du Développement durable, Serge Lepeltier, dont l'emploi du temps, occupé par plusieurs déplacements à l'étranger, ne lui a pas permis d'être parmi vous ce matin. Il m’a chargé néanmoins de vous dire tout l'intérêt qu'il portait aux travaux que vous conduisez aujourd'hui.

En effet, la problématique que vous abordez l'intéresse et intéresse particulièrement les services du ministère de l'Écologie et du Développement durable, tant l'ensemble des politiques relatives à la préservation de nos patrimoines que nous conduisons, qu'ils soient naturels ou paysagers, ont besoin d'être réinvesties d'un sens. Nous le voyons tous les jours, à l'issue, par exemple, pour le patrimoine naturel, de la conférence internationale sur la biodiversité.

En effet, si je crois qu'il n'y a plus de doute chez nos concitoyens sur l'appartenance au patrimoine des monuments historiques, depuis longtemps intégrés dans nos politiques et dans nos réactions, depuis Prosper Mérimée qui s'est battu parmi les premiers pour la reconnaissance de cette valeur, le patrimoine naturel et paysager dont s'occupe le ministre de l'Écologie et du Développement durable n'a pas encore acquis une véritable valeur, largement reconnue et partagée par nos concitoyens, ou pour le moins une valeur suffisante.

Le sujet que vous traitez aujourd'hui illustre parfaitement cette problématique.

Le chemin de fer, lorsque les premières lignes furent construites, a suscité parfois des oppositions farouches, à tel point que plusieurs villes ont refusé les gares, les fumées, le bruit, et certaines, qui étaient jusque-là dynamiques, ont progressivement, du fait de ce refus, vu leur développement s'inverser ou considérablement se ralentir. 
Nul doute, donc, que beaucoup des Français de la deuxième moitié du XIX ${ }^{e}$ siècle ont considéré alors la construction des lignes comme une dégradation de ce que nous appellerions aujourd'hui leur environnement. En même temps, cependant, le chemin de fer a apporté des possibilités de transports de marchandises, d'aller chercher des emplois au loin, de créer des emplois localement, en un mot des possibilités de développement économique et social extraordinaires.

Des débats ont été suscités à l'époque, auxquels font écho ceux que nous connaissons aujourd'hui à l'occasion de la création de nouvelles infrastructures. Le paysage et les valeurs que nous lui attachons constituent, en quelque sorte, un miroir qui nous permet de prendre du recul par rapport à nos débats et à nos pratiques actuelles; c'est la raison pour laquelle je pense que vos travaux sont pour nous d'un intérêt tout particulier.

Autrement dit, alors qu'aujourd'hui nous dénonçons comme une «balafre » dans un paysage l'autoroute ou, surtout, la ligne TGV qu'on veut y faire passer, ne deviendra-t-elle pas dans quelques temps un élément patrimonial ? C’est la question que je me suis posée au vu du programme de cette journée.

Je vous propose d'aborder ces questions sous deux aspects qui, d'expérience, me paraissent opérationnels : c'est en tout cas la manière dont un praticien les poserait aujourd'hui. Ces deux aspects sont le sens et la limite.

Le sens me parait une notion plus explicite que l'esthétique, voire que la notion de patrimoine, qui n'est pas forcément partagée par tous nos concitoyens, comme je le disais d'entrée.

C'est une notion plus facile à manier, utile pour se comprendre, parce qu'on peut en donner pour exemple des applications évidentes qui permettent d'entrer dans une discussion. Considérons par exemple les sites de la cathédrale de Chartres ou de la cathédrale d'Amiens, ou le site de Sénanque. On pourra en trouver beaucoup d'autres, moins prestigieux, comme une chapelle romane dans un environnement naturel qui n'a pas été altéré. Ces sites expriment, ces monuments expriment dans leur site quelque chose d'immédiatement évident qu'on n'arrive pas à rendre avec les mots ou que les mots ne rendent en général que maladroitement. Les mots que nous employons aujourd'hui sont sans doute très différents de ceux que pouvaient utiliser leurs bâtisseurs, tout autant que ce qu'on pouvait dire en ce XIX ${ }^{\mathrm{e}}$ siècle qui a vu la prise de conscience de l'intérêt des monuments historiques. 
Si leur sens originel échappe à ce jour à beaucoup d'entre nous, notamment lorsqu'il s'agit par exemple d'un monument spirituel dans un site urbain, il demeure cependant que ce rapport d'un monument protégé à son site est porteur d'une grande force qui nous parle encore avec évidence.

C'est la perception de ce sens qui nous anime dans la volonté de protéger les paysages ou du moins, sinon les protéger, les gérer d'une façon beaucoup plus attentive que ce qui a été fait auparavant. Dans ce rapport du joyau à l'écrin c'est finalement l'écrin, le site, qui donne dans sa dimension géographique un sens au monument.

Quel est alors le sens dont l'infrastructure ferroviaire est porteuse et qui lui donne aujourd'hui, à nos yeux, une valeur patrimoniale ? Bien qu'elle soit venue perturber les paysages ruraux traditionnels en y inscrivant des tracés radicalement nouveaux, une géométrie étrangère aux formes anciennes, nous avons progressivement réinvesti cette ligne, nous, hommes du début du xxI siècle, d'une valeur liée au voyage, au déplacement, à cette liberté de mouvement dont on ne jouissait pas auparavant, mais aussi à des valeurs de connexion à un réseau, à la possibilité de communication, d'échange, au lien qu'elle crée.

Enfin, l'infrastructure ferroviaire est porteuse des valeurs architecturales et de mémoire que je prenais comme exemple à l'instant mais aussi d'un témoignage des techniques, autant que des goûts artistiques, qui ont participé à la conception et au dessin des gares, des ouvrages d'art, en particulier des viaducs. Elle a marqué le paysage urbain de traces fortement structurantes qui fixent dans nos villes une étape bien datée de leur développement.

Cet ensemble d'éléments nous fait considérer que le patrimoine ferroviaire est autre chose qu'un simple système d'équipements techniques, qu'il revêt un caractère patrimonial et est porteur de sens et de valeur. En ce sens, votre démarche étend au domaine ferroviaire des démarches similaires et plus larges engagées depuis trente ans dans le domaine du patrimoine industriel.

Le deuxième éclairage que je souhaite jeter sur le début de cette journée est ce que j'ai appelé la limite. S'agissant d'ouvrages linéaires qui opèrent nécessairement une forte rupture, immédiatement lisible dans le paysage existant, cette notion me parait être un élément à explorer, notamment dans la perspective des ouvrages de notre temps. Il s'agit d'examiner de quelle manière la réflexion sur ces ouvrages perturbateurs du passé, aujourd'hui élevés au rang de patrimoine, peut nous aider dans notre avenir, dans la gestion de ces nouveaux équipements que nous continuons à produire. 
Les lignes TGV imposent en effet au territoire une logique radicalement nouvelle par rapport à celle des lignes ferroviaires classiques antérieures. Les concepteurs du TGV Est avec lesquels j’ai été amené à travailler m'ont ainsi expliqué que le train traditionnel empruntait les vallées et s'arrêtait dans toutes les villes, suivant le maillage urbain existant, alors qu'au contraire le TGV évite systématiquement les agglomérations, passe sur les plateaux et, donc, crée, investit des paysages, des milieux naturels qui jusqu'à présent n'avaient pas encore été "touchés » par l'infrastructure de transport moderne. De plus, cette ligne n'arrive pas seule, on le voit par exemple avec le TGV Est, elle s'accompagne d'ouvrages électriques importants, puisque le mode de consommation d'énergie du TGV exige des amenées de courant relativement fréquentes et de très forte puissance.

Les réflexions que vous menez aujourd'hui me paraissent donc pouvoir nous éclairer sur deux points.

Le premier apport consiste dans le recul que nous pouvons prendre dans les débats actuels pour savoir reconnaitre la structure qu'apporte un ouvrage ferroviaire à un paysage et apprendre à voir, pourquoi pas, comment elle l'enrichit.

Il s'agit pour nous, tenants de la protection du paysage, de nous débarrasser du réflexe qui consiste à vouloir systématiquement estomper, cacher et, au contraire, oser faire voir l'infrastructure, faire œuvre architecturale et paysagère et se servir de l'ouvrage et, au-delà, de la ligne pour prendre en main l'évolution d'un paysage, le faire évoluer, fixer de nouvelles limites, le hiérarchiser, le restructurer.

Réciproquement, c'est le deuxième point, vous pouvez nous aider à décider à quel moment un paysage doit être préservé. En effet, depuis la création des lignes au $\mathrm{XIX}^{\mathrm{e}}$ et au début du $\mathrm{Xx}^{\mathrm{e}}$ siècle, un facteur radicalement nouveau est intervenu : notre territoire national est de plus en plus labouré, coupé, tronçonné en petits morceaux par des infrastructures variées et diverses. Leurs effets sur le paysage n'a pas été évalué, surtout l'effet de répétition.

De même, si on prend le paysage au sens d'entité biologique, ce découpage opéré dans les milieux de vie et les connexions entre milieux encourage une tendance à l'isolement progressif des populations d'oiseaux, de la faune, voire de fleurs sauvages et contribue pour sa part à la perte de biodiversité que nous connaissons. Le risque de voir se généraliser un « mitage » du territoire et du paysage, la juxtaposition inorganisée d'éléments hétéroclites est en ce début du XXI siècle beaucoup plus réel qu'il ne pouvait l'être à la fin du XIx ${ }^{e}$ ou au début du $x^{e}$ siècle, surtout en milieu rural. 
Dans certains cas, alors, éviter de toucher à un paysage, estomper, gommer la trace de l'infrastructure dans le paysage peuvent aussi être une stratégie que l'on peut aller jusqu'à adopter en connaissance de cause, dans des cas très particuliers.

Nous attendons donc de vos réflexions un enrichissement de nos pratiques, étant entendu que le ministère de l'Écologie et du Développement durable reste attaché à une démarche d'accompagnement du paysage en mouvement, en évolution. Sa position fondamentale consiste à aborder la protection du paysage, y compris l'approche patrimoniale du paysage, dans une dynamique. Les outils à notre disposition pour soutenir cette position sont une sérieuse étude d'impact et, au-delà, une étude d'inscription paysagère des ouvrages très approfondie, en invitant les praticiens aujourd'hui, par votre journée d'études, à une réflexion sur la qualité patrimoniale des ouvrages. Imaginons que ces ouvrages, conçus d'abord comme des objets industriels, des prouesses d'ingénieurs, soient immédiatement un élément patrimonial par l'exigence de qualité de la démarche paysagère qui les aura accompagnés. Quels que soient les progrès considérables qui ont été accomplis en la matière par les maitres d'ouvrage depuis une vingtaine d'années, ils restent à généraliser.

Vous allez aider par vos réflexions nos concepteurs et ingénieurs à retrouver, grâce au dialogue aujourd'hui ouvert avec des historiens, des géographes, des sociologues, des paysagistes, des architectes, le fil de la tradition séculaire de ce métier, dont le symbole reste pour moi les dessins d'ingénieurs des Ponts et Chaussées de la fin du XviII siècle. Ils avaient atteint une qualité graphique et picturale extraordinaire qui exprimait bien la richesse de la démarche créative qu'ils avaient intégrée dans tous ses aspects. On a pu avoir l'impression que les ingénieurs du $\mathrm{xx}^{\mathrm{e}}$ siècle avaient progressivement oublié cette recherche de qualité. Je crois que les démarches dont je parlais et les journées de réflexion telles que celle-ci nous permettent de réinvestir leur métier de cette dimension qu'ils sont en train de retrouver, par cette attention portée à l'ouvrage, à sa qualité, à son sens, à sa dimension patrimoniale et paysagère. Soyez-en remerciés.

Je vous souhaite un excellent travail. 


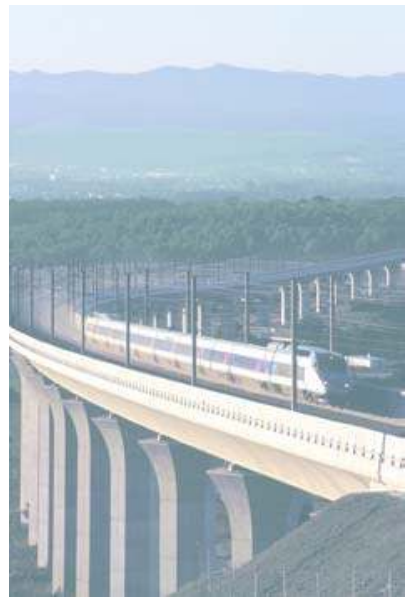

